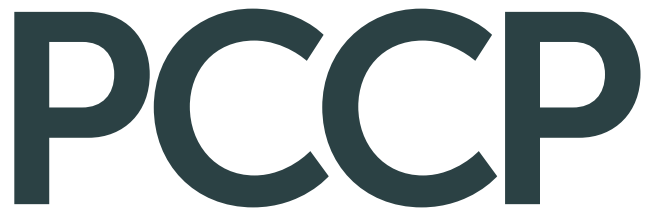

Physical Chemistry Chemical Physics www.rsc.org/pccp
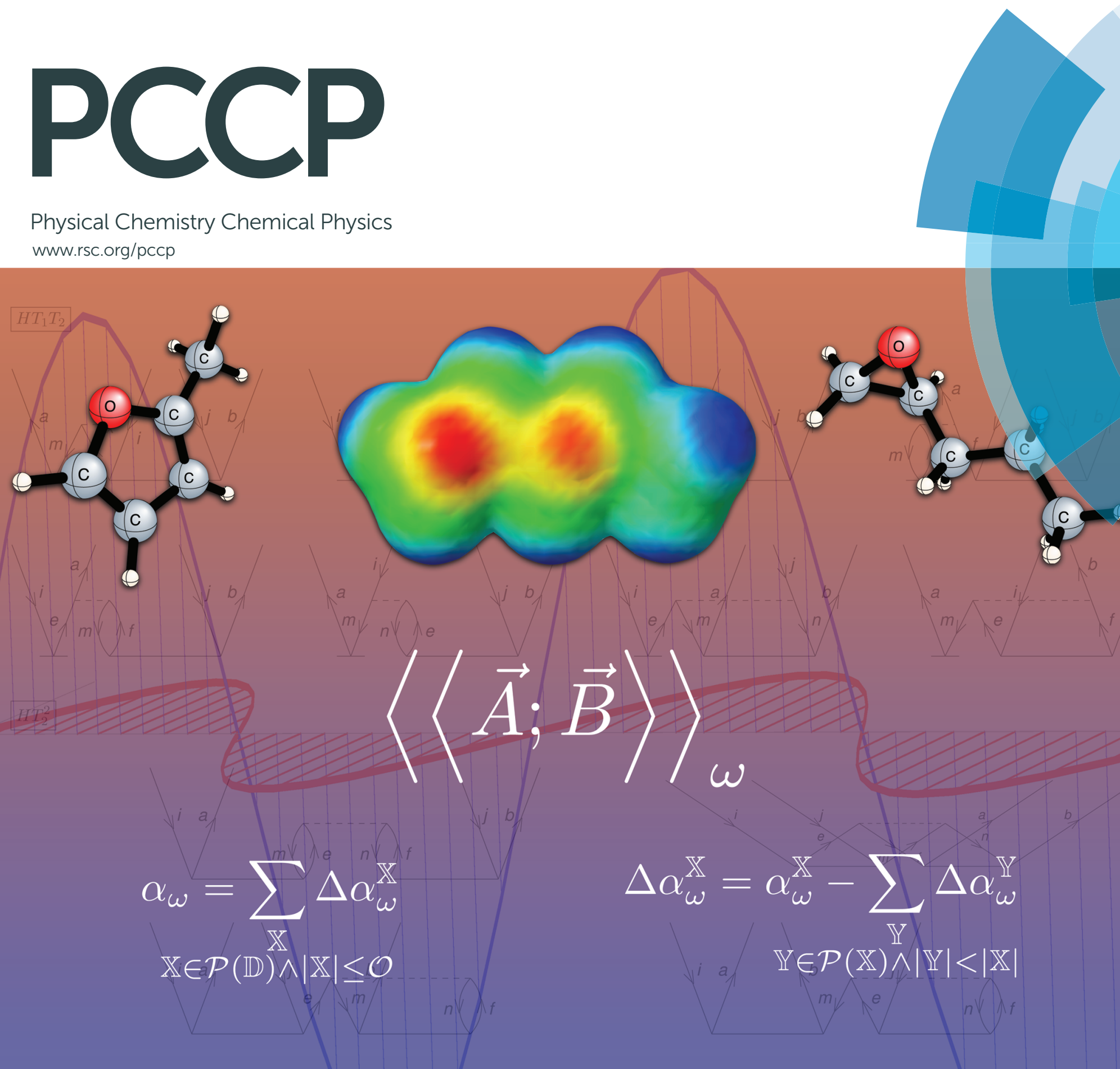

Themed issue: Theoretical chemistry developments: from electronic structure to simulations

ISSN 1463-9076 


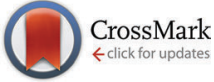

Cite this: Phys. Chem. Chem. Phys., 2015, 17, 14284

Received 3rd November 2014 Accepted 8th December 2014 DOI: $10.1039 / c 4 c p 05076 b$

www.rsc.org/pccp

\title{
Incremental evaluation of coupled cluster dipole polarizabilities $\dagger$
}

\author{
Joachim Friedrich, ${ }^{* a}$ Harley R. McAlexander, ${ }^{\mathrm{b}}$ Ashutosh Kumar ${ }^{\mathrm{b}}$ and \\ T. Daniel Crawford ${ }^{b}$
}

\begin{abstract}
In this work we present the first implementation of the incremental scheme for coupled cluster linearresponse frequency-dependent dipole polarizabilities. The implementation is fully automated and makes use of the domain-specific basis set approach. The accuracy of the approach is determined on the basis of a test suite of $\mathbf{4 7}$ molecules and small clusters. The local approximation in the coupled cluster singles and doubles polarizability exhibits a mean error of $0.02 \%$ and a standard deviation of $0.32 \%$ when using a third-order incremental expansion. With the proposed approach, it is possible to compute polarizabilities with larger basis sets compared to the canonical implementation and thus it is possible to obtain higher total accuracy. The incremental scheme yields the smallest errors for weakly-bound and quasi-linear systems, while two- and three-dimensional (cage-like) structures exhibit somewhat larger errors as compared to the full test set.
\end{abstract}

\section{Introduction}

Quantum chemical methods such as coupled cluster theory ${ }^{1-4}$ can provide robust and reliable simulations of both resonant and non-resonant interactions of molecules with electric and magnetic fields. ${ }^{5}$ The coupled cluster singles and doubles (CCSD) method, ${ }^{6}$ as well as CCSD augmented with approximate triples [e.g., $\operatorname{CCSD}(\mathrm{T})$ or $\mathrm{CC} 3],{ }^{7,8}$ have been found to provide very high accuracy in simulations of UV/vis ${ }^{9-14}$ and circular dichroism spectra $^{15-19}$ as well as dipole (hyper)polarizabilities, magnetizabilities, or optical rotations. ${ }^{17-25}$

Unfortunately, conventional coupled cluster methods based on canonical molecular orbitals (MOs) are limited to relatively small molecules and clusters (containing $c a .20$ non-hydrogen atoms, in the absence of symmetry) both due to their slow convergence with respect to the one-particle basis set ${ }^{26}$ and their high-degree polynomial scaling- $\mathcal{O}\left(N^{6}\right)$ for CCSD and at least $\mathcal{O}\left(\mathrm{N}^{7}\right)$ for methods that include triples. The former typically requires basis functions with high levels of angular momentum for precise computations near the complete-basis-set (CBS) limit, though the problem can be reduced somewhat though CBS extrapolation ${ }^{27}$ or by introducing terms into the wave function that include the electron-electron distance explicitly. ${ }^{28,29}$

\footnotetext{
${ }^{a}$ Institute for Chemistry, Chemnitz University of Technology, Straße der Nationen 62, 09111 Chemnitz, Germany. E-mail: joachim.friedrich@chemie.tu-chemnitz.de

${ }^{b}$ Department of Chemistry, Virginia Tech, Blacksburg, Virginia 24061, USA

$\dagger$ Electronic supplementary information (ESI) available: The supplementary material contains the Cartesian coordinates for all 47 test molecules, as well as incremental CCSD energies, polarizabilities, and corresponding errors. See DOI: 10.1039/c4cp05076b
}

The polynomial scaling wall was first addressed by the pioneering efforts of Pulay and Saebø in the development of the local correlation framework. ${ }^{30,31}$ By adopting a more localized representation of the occupied and virtual MO spaces, one can take advantage of the resulting sparsity in the wave function, reducing the number of parameters/amplitudes that must be computed and stored. The Pulay-Saebø approach has been utilized with great success by Werner, Schütz, and co-workers, for example, who have extended the applicability of coupled cluster methods to systems containing more than a hundred atoms. ${ }^{32-38}$ Alternative local correlation approaches in the same spirit include the orbital-specific virtual idea of Yang et $a .^{39}$ and the local pair-natural-orbital (LPNO) approach recently reintroduced by Neese and co-workers. ${ }^{4-42}$ Other methods make use of a fragmentation approach in which a larger system is partitioned into smaller components, such as the fragment-MO approach of Fedorov, Kitaura, Gordon and co-workers, ${ }^{43-47}$ divideand-conquer scheme ${ }^{48-50}$ the natural linear scaling coupled cluster $^{51-53}$ or the cluster-in-molecule method (CIM). ${ }^{54-60}$ (For a recent review of fragmentation methods, see ref. 61.)

While the development of fast coupled cluster methods to compute highly accurate energies of large systems has seen great success in the last few years, the computation of many other properties has advanced more slowly. Within the Pulay-Saebø framework, Werner, Schütz, Korona, and co-workers ${ }^{62-64}$ have carried out locally correlation coupled cluster computations of dipole moments, static polarizabilities, and excitation energies at the CCSD level, while Schütz and co-workers have reported local second-order CC (CC2) computations of transition properties, excitation energies, and excited-state dipole moments. ${ }^{65-67}$ 
Crawford and co-workers have also utilized the Pulay-Saebø approach in the computation of dynamic response functions, including excitation energies, ${ }^{68}$ frequency-dependent polarizabilities, ${ }^{69}$ and optical rotations. ${ }^{70-72}$

In this work we apply the incremental scheme of Stoll ${ }^{73-75}$ to compute the CCSD frequency-dependent dipole polarizabilities. The incremental scheme is a generalization of the Bethe-Goldstone expansion and was introduced to quantum chemistry by Nesbet. ${ }^{76-78}$ In this method the system is divided into domains of localized occupied orbitals. The total energy of the system is obtained by computing the correlation energies of single domains, of domain pairs, of domain triples, etc. until the desired accuracy is obtained. This method was successfully applied to compute coupled cluster energies in various applications for periodic systems, ${ }^{79-90}$ to closed- ${ }^{91-98}$ and open-shell molecular systems, ${ }^{99,100}$ and recently also to metals with a multireference wavefunction. ${ }^{101}$ In the context of properties, Yang and Dolg computed static first- and second-order polarizabilities $^{102}$ and Friedrich et al. computed dipole and quadrupole moments. ${ }^{103}$ In this work we implemented the incremental CCSD polarizabilities within the PSI4 program package using the domain-specific basis set approach to reduce the computational effort. The performance of the incremental scheme for CCSD polarizabilities is analyzed for a set of 47 molecules using the cc-pVDZ basis set and for 23 using the aug-cc-pVDZ basis set.

Finally we note that the errors provided in this work are solely those of the local approximations. The small double- $\zeta$ basis sets applied in this work introduce a significant error in the dipole-polarizability and therefore it is necessary to use significantly larger basis sets in production calculations. ${ }^{104}$ When attempting to achieve high accuracy coupled cluster calculations, it is also necessary to include higher excitations in the calculations of the polarizabilities. ${ }^{105}$

\section{Theory}

In coupled cluster response theory, ${ }^{5,106}$ the linear response function associated with dynamic perturbations $\vec{A}$ and $\vec{B}$ is the second derivative of the coupled cluster time-averaged Lagrangian functional (quasi-energy),

$$
\begin{aligned}
\langle\langle\vec{A} ; \vec{B}\rangle\rangle_{\omega}= & \frac{\mathrm{d}^{2}\left\{L_{\mathrm{CC}}\right\}_{t}}{\mathrm{~d} \vec{A} \mathrm{~d} \vec{B}} \\
= & \frac{1}{2} \hat{C}^{ \pm \omega} \hat{P}[A(-\omega), B(+\omega)] \\
& \times\left[\left\langle 0\left|\Lambda\left[\bar{A}, X_{\omega}^{B}\right]\right| 0\right\rangle+\frac{1}{2}\left\langle 0\left|\Lambda\left[\left[\bar{H}, X_{-\omega}^{A}\right], X_{\omega}^{B}\right]\right| 0\right\rangle\right] .
\end{aligned}
$$

In this expression, $|0\rangle$ is the reference determinant, the overbar denotes similarity transformation of the given operator, e.g.,

$$
\bar{H}=\mathrm{e}^{-T} H \mathrm{e}^{T},
$$

where $T$ is the ground-state cluster operator, and $\Lambda$ is the de-excitation cluster operator associated with the left-hand wave function. The permutation operator, $\hat{P}$, symmetrizes the expression with respect to the perturbations and $\hat{C}^{ \pm \omega}$ symmetrizes with respect to complex conjugation and sign reversal of the frequency, $\omega$. The first-order perturbed wave functions with respect to perturbation $A, X_{\omega}^{A}$, are obtained by solving the systems of linear equations,

$$
\left\langle\phi\left|(\bar{H}-\omega) X_{\omega}^{A}\right| 0\right\rangle=-\langle\phi|\bar{A}| 0\rangle .
$$

If the operators $\vec{A}$ and $\vec{B}$ are both taken to be the electric-dipole operator (which can be cast in either the length or velocity representations), ${ }^{107}$ the frequency-dependent dipole-polarizability tensor, $\alpha_{\omega}$, is the negative of the resulting linear-response function

$$
\alpha_{\omega}=-\langle\langle\mu ; \mu\rangle\rangle_{\omega} .
$$

In conventional calculations of the coupled cluster linear response function, contributions arising from the relaxation of the reference MOs (typically Hartree-Fock orbitals) are neglected in order to preserve the (first-order) pole structure of the response function as the field frequency approaches a resonance. The MO response is typically small for such properties and accounted for in large part by the inclusion of the single-excitation operator in the cluster expansion. ${ }^{108}$

The incremental scheme is a many-body approach where the total system is partitioned into disjoint sets of localized occupied orbitals, referred to as one-site domains. ${ }^{109-111}$ In this scheme only the correlation part of the energy is partitioned, and the total energy of the system is computed by: ${ }^{73,109}$

$$
E_{\text {corr }}=\sum_{\substack{\mathbb{X} \\ \mathbb{X} \in \mathcal{P}(\mathbb{D}) \wedge|\mathbb{X}| \leq \mathcal{O}}} \Delta \varepsilon^{\mathbb{X}}
$$

where $\mathcal{P}(\mathbb{D})$ is the power set of the set of the domains $\mathbb{D}$. The restriction to the cardinality of the sets $\mathbb{X}$ truncates the incremental series at the desired order $\mathcal{O}$. The general increment is defined as:

$$
\Delta \varepsilon^{\mathbb{X}}=\varepsilon^{\mathbb{X}}-\sum_{\mathbb{Y} \in \mathcal{P}(\mathbb{X}) \wedge|\mathbb{Y}|<|\mathbb{X}|} \Delta \varepsilon^{\mathbb{Y}}
$$

where $\varepsilon^{\mathbb{X}}$ is the correlation energy of the combined subsystems of $\mathbb{X}$. Properties that may be expressed as (quasi)energy derivatives, such as the frequency-dependent dipole-polarizability, may be calculated in exactly the same manner as the energy:

$$
\alpha_{\omega}=\sum_{\substack{\mathbb{X} \\ \mathbb{X} \in \mathcal{P}(\mathbb{D}) \wedge|\mathbb{X}| \leq \mathcal{O}}} \Delta \alpha_{\omega}^{\mathbb{X}}
$$

and

$$
\Delta \alpha_{\omega}^{\mathbb{X}}=\alpha_{\omega}^{\mathbb{X}}-\sum_{\mathbb{Y} \in \mathcal{P}(\mathbb{X}) \wedge|\mathbb{Y}|<|\mathbb{X}|} \Delta \alpha_{\omega}^{\mathbb{Y}}
$$

where $\alpha_{\omega}^{\mathbb{X}}$ is the polarizability of the combined subsystems of $\mathbb{X}$. Furthermore this task is somewhat simplified in the case of response properties, in that orbital relaxation contributions are excluded, as noted above.

The computation can be further simplified by reducing the virtual space in each partition. This can be accomplished via the domain-specific basis set approximation, within which we classify the atoms into a main region and its environment using 


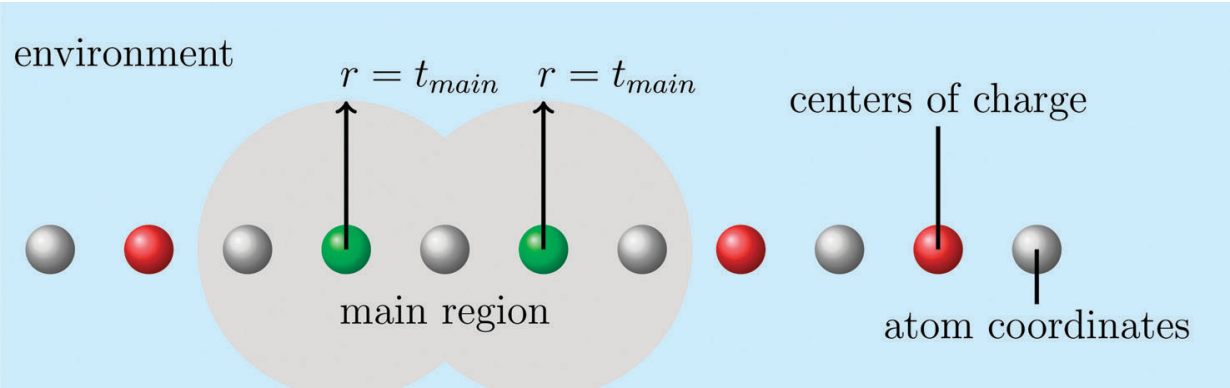

Fig. 1 Construction of the domain-specific basis set. The centers of charge of a domain are given in green, and the most important region of a domain is given by the grey area. The area is defined by the union of the circles with the radius $t_{\text {main }}$ around every center of charge in a domain (green circles). The large basis set is applied in the main region and the small basis set in the rest of the system.

the parameter $t_{\text {main }}$ (see Fig. 1). With this procedure we select all atoms close to the occupied orbitals in the domain as important and thus treat them with a large basis set, whereas all atoms in the environment are treated with a small double- $\zeta$ basis set without polarization functions. After the construction of the domain-specific basis set we generate orthogonal localized occupied orbitals in this basis set by a Hartree-Fock calculation and a subsequent Boys localization. ${ }^{112}$

Finally, a distance screening cutoff may be employed to achieve linear scaling in the number of domain calculations

Table 1 Structures of the molecules in the chosen test set (part I). The letters refer to the subsets used for the further analysis. The molecules marked with an s belong to the subset used in the analysis of the basis set error. The $\mathrm{w}$ refers to the subset of weakly interacting systems, the I to the linear molecules and the $\mathrm{nl}$ to the non-linear molecules

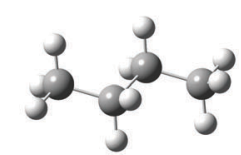

$1, \mathrm{~s}, 1$

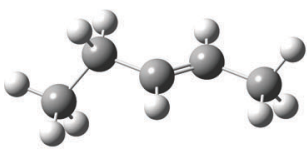

$5, \mathrm{~s}, 1$

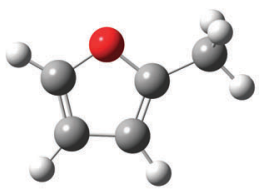

$9, \mathrm{~s}, \mathrm{nl}$

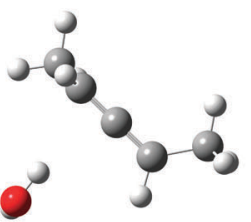

$13, \mathrm{w}$

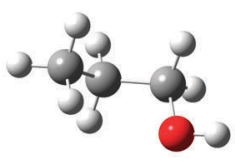

$2, \mathrm{~s}, 1$

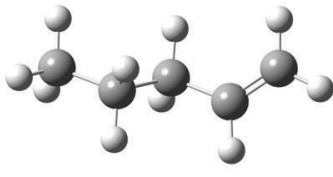

$$
6, \mathrm{~s}, 1
$$

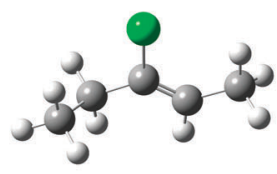

$10, \mathrm{~s}, 1$

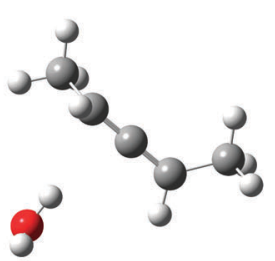

$14, \mathrm{w}$

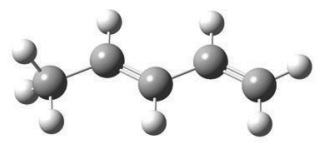

$3, \mathrm{~s}, 1$

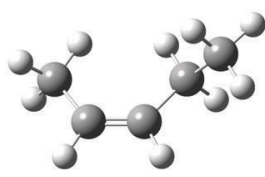

$7, \mathrm{~s}, 1$

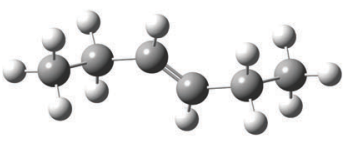

11,1

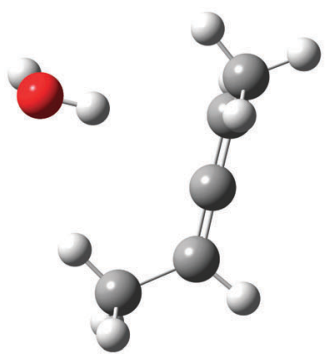

$15, \mathrm{~s}, \mathrm{w}$

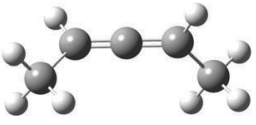

$4, \mathrm{~s}, 1$

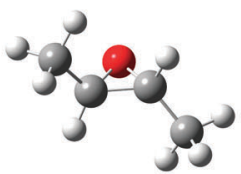

$$
8, \mathrm{~s}, 1
$$

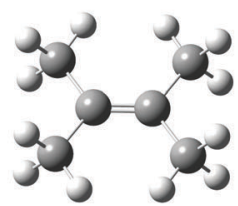

$12, \mathrm{~s}, \mathrm{nl}$

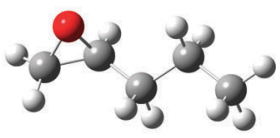

$16, \mathrm{~s}, 1$ 
Table 2 Structures of the molecules in the chosen test set (part II). The letters refer to the subsets used for the further analysis. The molecules marked with an s belong to the subset used in the analysis of the basis set error. The $w$ refers to the subset of weakly interacting systems, the I to the linear molecules and the $\mathrm{nl}$ to the non-linear molecules

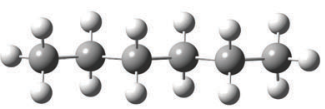

$17, \mathrm{~s}, 1$

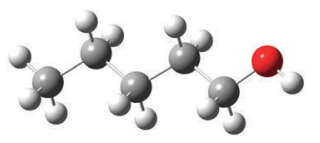

21,1

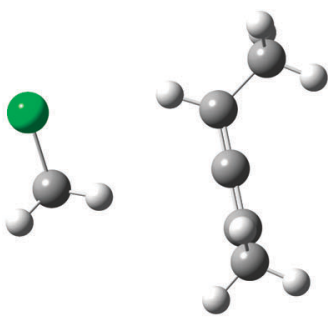

$25, \mathrm{w}$

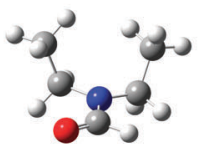

$29, \mathrm{nl}$

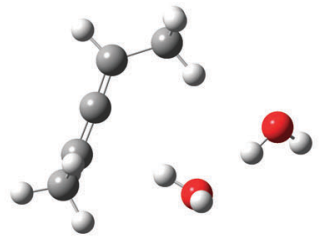

$33, \mathrm{w}$

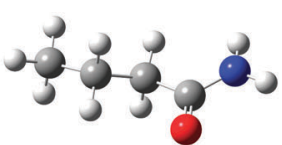

$18, \mathrm{~s}, 1$

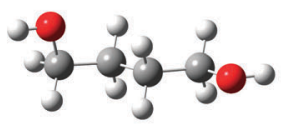

$22, \mathrm{~s}, 1$

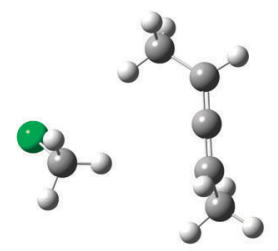

$26, \mathrm{w}$

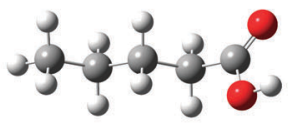

30,s,1

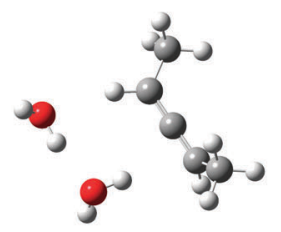

$34, \mathrm{w}$

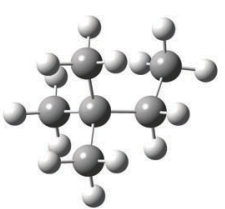

19,s,nl

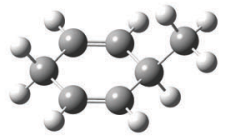

23,s,nl

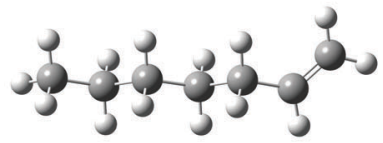

27,s,1

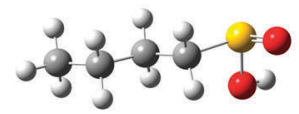

31,s,1

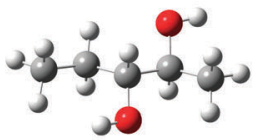

35,1

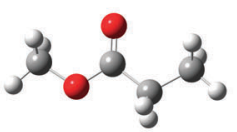

$20, \mathrm{~s}, 1$

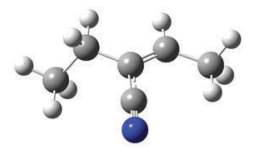

24,nl
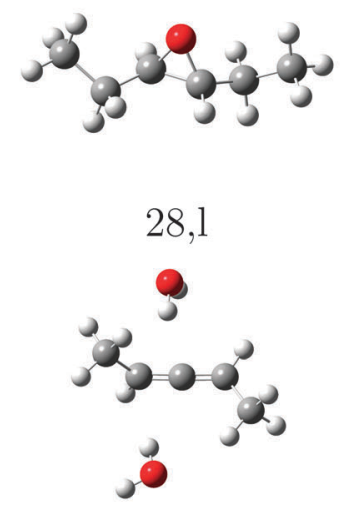

$32, \mathrm{w}$ with increasing the size of the system. Considering eqn (5) the number of calculations $\mathscr{N}$ grows with

$$
\mathcal{N}=\sum_{i=1}^{\mathcal{O}}\left(\begin{array}{c}
|\mathbb{D}| \\
i
\end{array}\right)
$$

However it is not necessary to compute all increments, since the increments decay with increasing order and with the spatial distance of the underlying domains. We account for this by applying an order-dependent truncation threshold $t_{\text {dist }}(i)=f(i-1)^{2}$, where $i$ is the index of the order and $f$ an adjustable parameter (vide infra). ${ }^{110,113}$

\section{Computational details}

In order to test the incremental scheme's performance for dynamic polarizabilities, a test set of 47 molecules and clusters was selected containing both first row $(\mathrm{C}, \mathrm{O}, \mathrm{N})$ and second 
Table 3 Structures of the molecules in the chosen test set (part III). The letters refer to the subsets used for the further analysis. The molecules marked with an s belong to the subset used in the analysis of the basis set error. The $\mathrm{w}$ refers to the subset of weakly interacting systems, the I to the linear molecules and the $\mathrm{nl}$ to the non-linear molecules

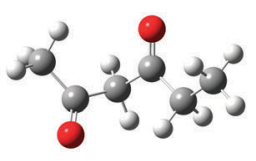

37,1

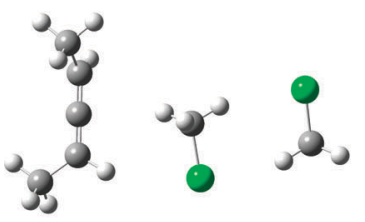

$41, \mathrm{w}$

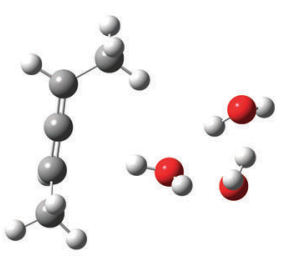

$45, \mathrm{~W}$

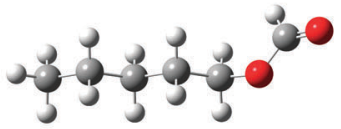

38,1

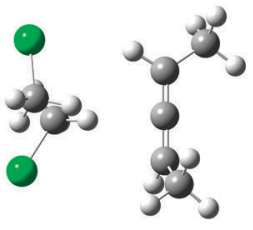

$42, \mathrm{w}$

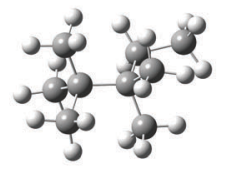

$46, \mathrm{nl}$

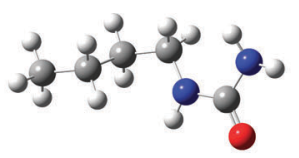

39,1

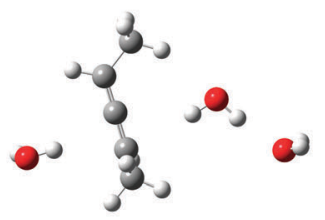

$43, \mathrm{w}$

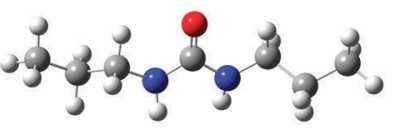

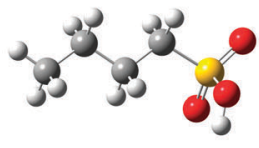

$40, \mathrm{~s}, 1$

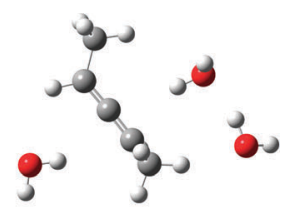

$44, \mathrm{w}$ row $(\mathrm{S}, \mathrm{Cl})$ elements. The systems are shown in Tables 1-3 and are ordered according to the increasing magnitude of the CCSD correlation energy. The Cartesian coordinates of each structure, the incremental CCSD and MP2 energies, and the corresponding errors are given in the ESI. $\dagger$ Most of the structures were optimized at the BP86/def2-TZVP ${ }^{114-117}$ level of theory, as reported in ref. 118, and structures 4, 13-15, 25, $26,32-34,41-45$ were optimized at the B3LYP/aug-cc-pVDZ level of theory. ${ }^{119-124}$

The partitioning of the occupied space was carried with a fully automatic black box scheme, ${ }^{118}$ in conjunction with coupled cluster calculations performed with a development version of the PSI4 program package. ${ }^{125}$ The truncation parameters ${ }^{113,118,126}$ in the incremental series are $t_{\text {main }}=3$ Bohr, the $\mathrm{dsp}=4$ and $f=30$. dsp is a measure for the number of occupied orbitals in a domain, i.e. it controls the size of the domain. The main region of each increment was treated with the cc-pVDZ basis set for the large test set and with the aug-cc-pVDZ basis set in smaller subset. In the environment we applied the 6-31G basis $\operatorname{set}^{127}$ in all calculations. The required increment data such as MO-coefficients, overlap and dipole integrals was obtained via an interface to the PSI4 code. Core orbitals (1s for $\mathrm{C}, \mathrm{N}$, and $\mathrm{O} ; 1 \mathrm{~s} 2 \mathrm{~s} 2 \mathrm{p}$ for $\mathrm{S}$ and $\mathrm{Cl}$ ) were frozen in all reference and increment computations. Hardware limitations prevented computation of reference CCSD polarizabilities in the aug-cc-pVDZ basis set for some cases, and thus a subset of 23 structure $(1-10,12,15-20,22,23,27,30,31,40)$ was used to determine basis set effects. In order to perform an analysis of the errors $\Delta_{i}=E_{i}^{\text {approx }}-E_{i}^{\text {ref }}$ introduced by local approximations, we employed the following statistical measures:

$$
\begin{gathered}
\text { mean deviation: } \mu=\frac{1}{n} \sum_{i=1}^{n} \Delta_{i} \\
\text { standard deviation: } \sigma=\sqrt{\frac{1}{n-1} \sum_{i=1}^{n}\left(\Delta_{i}-\mu\right)^{2}} .
\end{gathered}
$$

For a graphical representation of the errors we assume a Gaussian distribution:

$$
G(x)=\frac{1}{\sigma \sqrt{2 \pi}} \mathrm{e}^{-\frac{1}{2}\left(\frac{x-\mu}{\sigma}\right)^{2}}
$$

\section{Results and discussion}

\subsection{Accuracy of dipole polarizabilities}

Although we computed the dipole polarizabilities for all 47 test systems across a range of wavelengths from 355 to $633 \mathrm{~nm}$, the errors due to the incremental expansion are very similar. Therefore we will focus the discussion primarily on the results obtained with $\lambda=589 \mathrm{~nm}$. In the left-hand plot of Fig. 2 we present the absolute and relative errors due to the incremental expansion in the right-hand plot. At second order, the maximum absolute error is 17 a.u. and the mean error is 0.5 a.u. At the third 

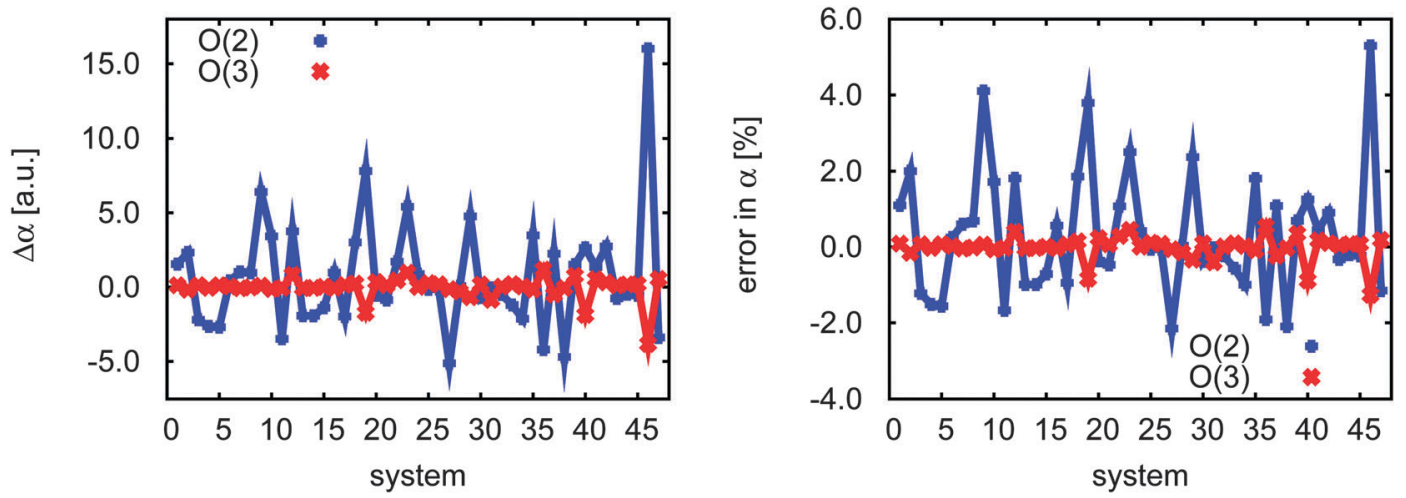

Fig. 2 Absolute errors (a.u., left) and relative errors (\%, right) in the CCSD/cc-pVDZ polarizabilities of the full test set of 47 systems, ordered by increasing correlation energy. Errors in the second- and third-order expansions are given in blue and red, respectively.

order both maximum absolute error and mean error decrease by nearly an order of magnitude to 3.0 a.u. and 0.06 a.u., respectively. The relative errors are plotted on the right-hand side of Fig. 2 and show the same pattern. The relative maximum absolute errors are around $5 \%$ at second order and around $1 \%$ at the third order expansion, whereas the mean error decreases from $0.25 \%$ at second order to only $0.02 \%$ at third order.

Based on the work of Hald et al. ${ }^{104}$ we expect a basis set error of a few \%, when going from the double to the triple- $\zeta$ level. From this perspective the incremental scheme in combination with the domain-specific basis set becomes very attractive, as it becomes more efficient in larger basis sets. Furthermore one can use the incremental scheme to reduce the total error in the calculation, since a larger basis set may be computationally affordable and the local approximations introduce only small errors (vide infra). Based on the results of Christiansen et al. ${ }^{105}$ it is evident that also the higher excitations may contribute to the polarizability up to a few $\%$. Since the errors of the local approximations are smaller on average, it is very promising to include higher excitations in the incremental property calculations.

Due to the computational demands of the CCSD method we were not able to obtain polarizabilities for all 47 test cases using the aug-cc-pVDZ basis set, and thus we considered a smaller set of 23 systems $(1-10,12,15-20,22,23,27,30,31,40)$ to investigate basis-set effects. As shown in Fig. 3, the error distributions in both second and third order are very similar for the small and large test sets, suggesting that the smaller group of molecules is sufficiently representative of the complete test set that it may be used to investigate basis set effects.

In Fig. 4 we present the errors in the CCSD dipole polarizabilities due to the incremental scheme using the 23-molecule test set obtained with the cc-pVDZ basis set (referred to as DZ and plotted in red) and with the aug-cc-pVDZ basis set (AVDZ, blue curves). In second order, the polarizabilities are slightly overestimated on average, and the addition of diffuse basis functions increases this error by $c a .0 .27 \%$. However, at third order, the opposite trend dominates, and a slight underestimation is observed as compared to the full canonical CCSD polarizabilities. Furthermore, diffuse functions increase this error by $0.14 \%$, but the error remains small in both orders.

Which approximation introduces the larger errors, the basis set or the order of truncation of the incremental scheme? In Fig. 5, the red Gaussian distribution gives the errors in the dipole polarizabilities obtained with the cc-pVDZ basis set as
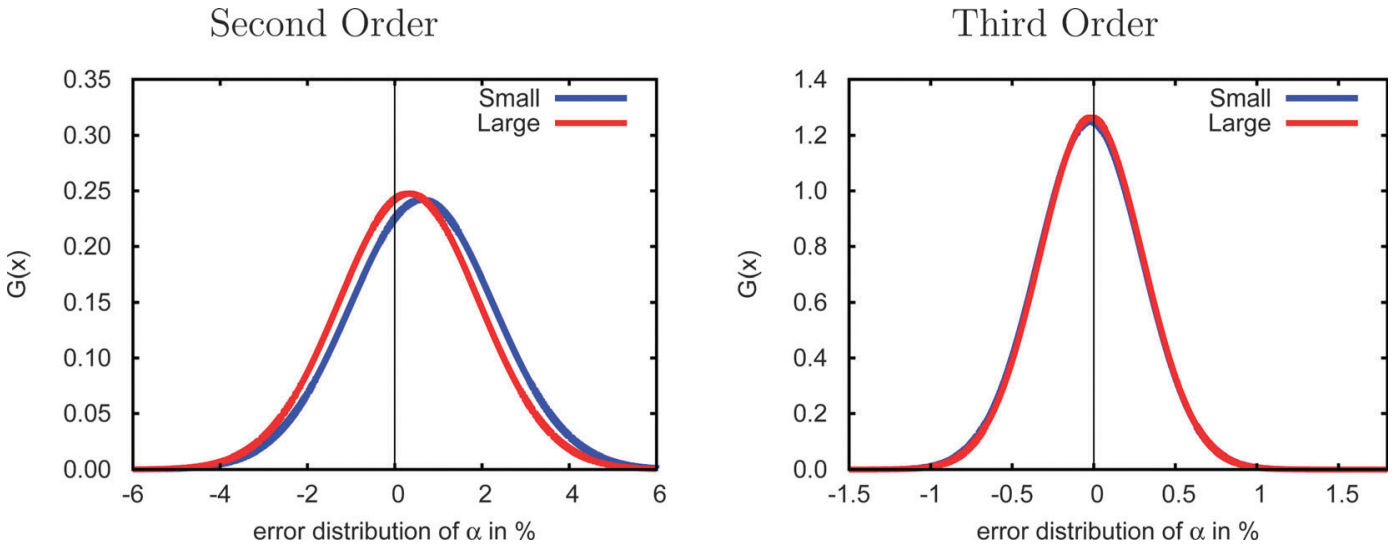

Fig. 3 Gaussian distributions of the errors in the second-order (left) and third-order (right) incremental scheme in the CCSD/cc-pVDZ polarizabilities for the full test set of 47 molecules and for a subset of 23 smaller molecules. The distributions are very similar for both test sets indicating that the smaller set may be used to study basis-set effects. 

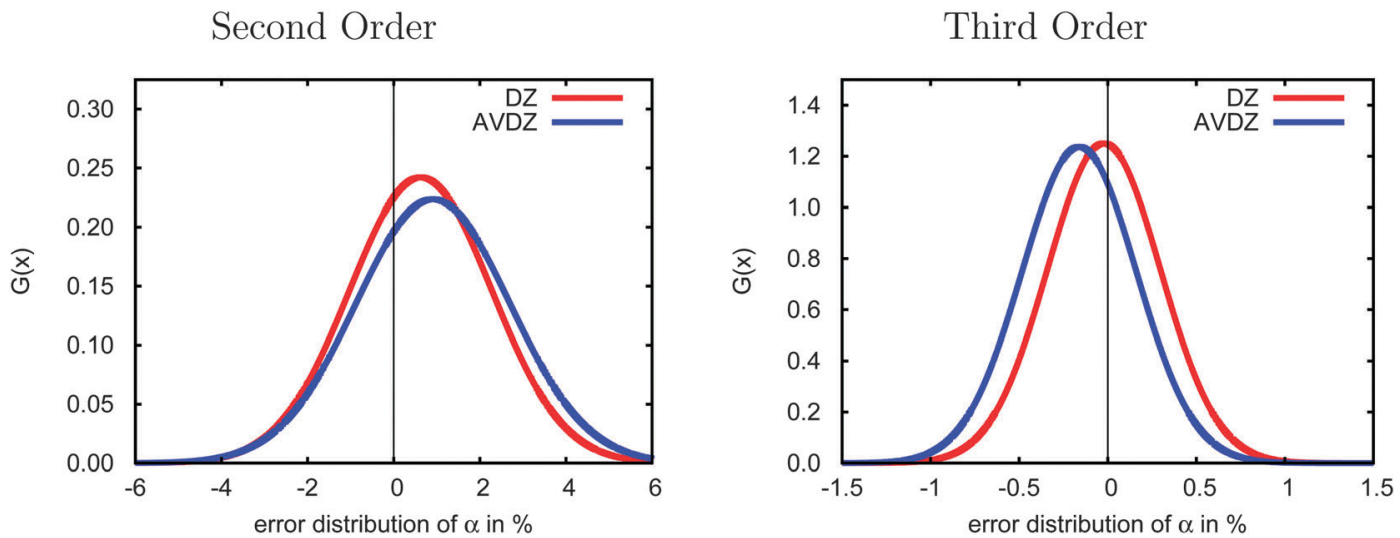

Fig. 4 Comparison of the errors due to the incremental expansion of the CCSD polarizabilities for the cc-pVDZ (DZ) and aug-cc-pVDZ (AVDZ) basis sets using Gaussian distributions for the small-molecule test set. The inclusion of diffuse basis functions shifts the second-order errors up by $0.27 \%$ and the third-order down by $0.14 \%$

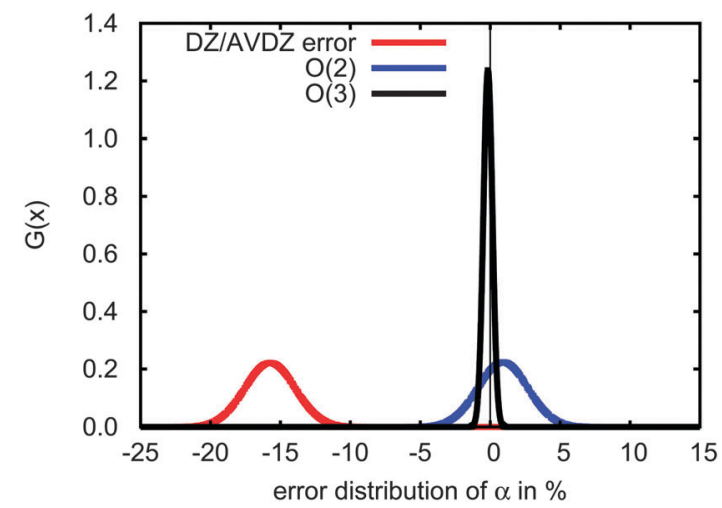

Fig. 5 Basis set dependence of the CCSD polarizability compared to the errors due to the key approximations in the incremental series using the subset of 23 molecules (vide supra). DZ/AVDZ refers to the deviation between the cc-pVDZ (DZ) and aug-cc-pVDZ (AVDZ) basis sets using the standard CCSD code. The $\mathrm{O}(2)$ and $\mathrm{O}(3)$ values correspond to secondand third-order incremental expansions, respectively, both using the aug$c c-p V D Z$ basis set. Please note that all errors are relative to the standard CCSD/aug-cc-pVDZ calculation. Without diffuse functions the polarizabilities are about $16 \%$ too low, and the standard deviation of the second order expansion is similar to the standard deviation due to the small basis set approximation. The third order expansion is significantly more accurate.

compared to the aug-cc-pVDZ basis set, while the blue and black distributions represent the errors in the second- and third-order expansions, respectively, obtained with the aug-ccpVDZ basis set. The basis-set truncation yields a mean error of approximately $16 \%$ due to the lack of diffuse functions, while even the second-order expansion produces a mean error of less than $2 \%$. Clearly a second-order expansion is acceptable, but the use of diffuse functions is essential.

Fig. 6 compares the errors in the correlation energy (red curve) to the errors in the dipole polarizabilities (blue) due to the incremental-scheme truncation at second order (top) and third order (bottom), with the data ordered according to increasing energy errors. In general the variation of the errors in the polarizability is higher than the variation in the energy, which is unsurprising given that energy depends only on the
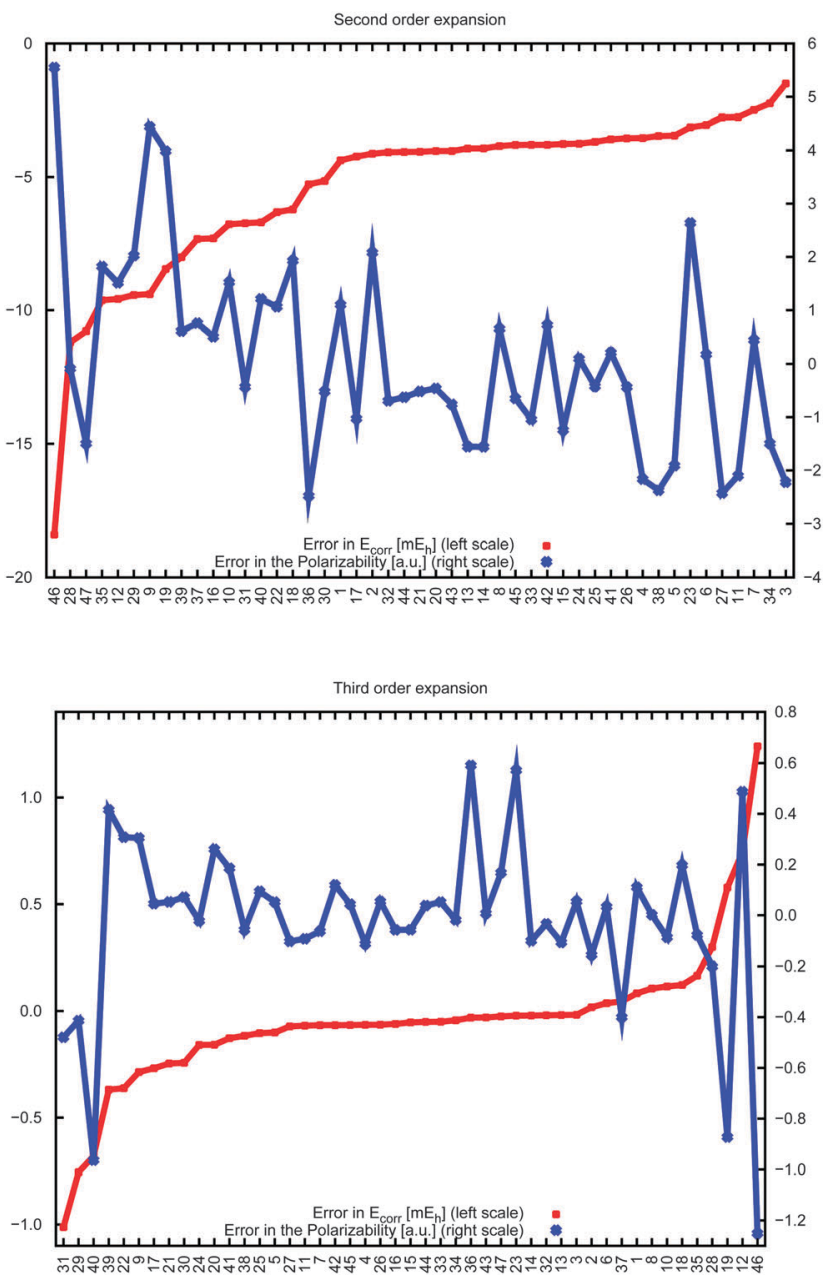

Fig. 6 Comparison of errors due to the incremental expansion of the CCSD/ $c c-p V D Z$ energies $v s$. polarizabilities. The results are ordered by increasing error of the correlation energy. (N.B.: the ordering of the molecules changes between the plots due to shifts in the errors in the energies.)

wave function, whereas linear-response properties are related to the derivative of the wave function with respect to an external 
field. For the third-order expansion, a large error in the energy clearly correlates to a large error in the polarizability, but no obvious systematic trends appear in the second-order expansion. In addition, there are select cases for which the energy is well reproduced by the incremental scheme but relatively large errors appear in the polarizability (vide infra). Therefore the errors in the energies cannot be used to estimate the corresponding accuracy of the polarizability.

\subsection{Corrections for basis set errors}

As noted above, omission of diffuse functions in the basis set yields CCSD/cc-pVDZ dipole polarizabilities that are roughly $16 \%$ too low as compared to the CCSD/aug-cc-pVDZ level. If this mean error $(\mu)$ is simply used to shift the polarizabilities, one obtains the distribution plotted in cyan in Fig. 7. However, we may devise an improved basis-set correction using lower orders in the incremental scheme. When using the domain-specific basis set in the incremental scheme, only a few atoms require the large basis set at low order. Furthermore, the computation of the higher-order terms requires by far the most CPU-time. Therefore it is very attractive to carry out the incremental expansion in a small basis set and estimate the basis set effect from low order terms with the large basis set. We may estimate the basis-set error,

$$
\Delta \alpha_{\omega}=\alpha_{\omega}(\text { cc-pVDZ })-\alpha_{\omega}(\text { aug-cc-pVDZ }),
$$

using a low-order calculation as

$$
\Delta_{1} \alpha_{\omega}=\sum_{\substack{\mathbb{X} \\ \mathbb{X} \in \mathcal{P}(\mathbb{D}) \wedge|\mathbb{X}|=1}}\left[\Delta \alpha_{\omega}^{\mathbb{X}}(\text { cc-pVDZ })-\Delta \alpha_{\omega}^{\mathbb{X}}(\text { aug-cc-pVDZ })\right]
$$

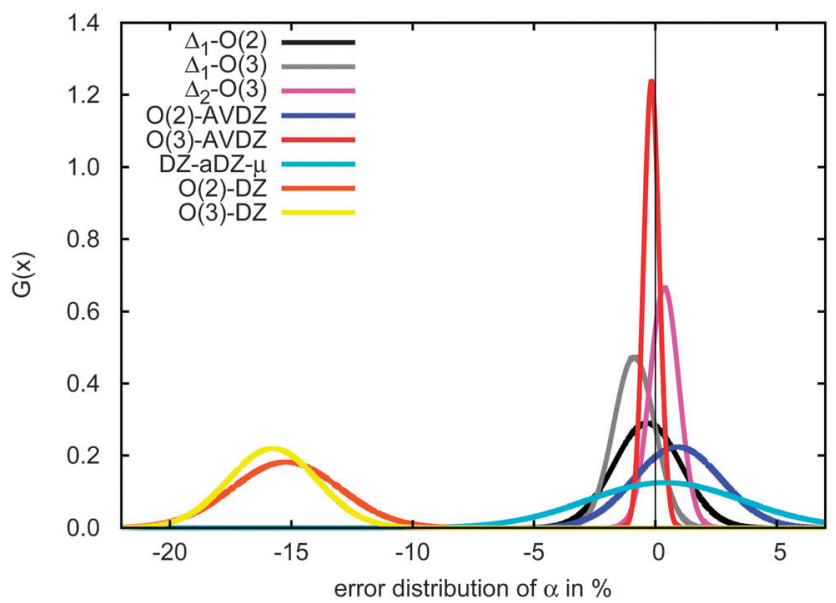

Fig. 7 Performance of simple methods to correct CCSD polarizabilities for basis set errors. Cyan: subtraction of the mean CCSD/cc-pVDZ basis set error; black: second-order incremental CCSD/cc-pVDZ expansion with a first-order basis-set correction [eqn (10)]; grey: third-order CCSD/ cc-pVDZ expansion and first-order basis-set correction [eqn (10)]; pink: third-order incremental expansion and second-order basis-set correction [eqn (11)]; blue: second-order incremental expansion in the large aug-ccpVDZ basis set; red: third-order incremental expansion in the large augcc-pVDZ basis set. Orange: uncorrected second-order incremental expansion; yellow: uncorrected third-order incremental expansion.

$$
\Delta_{2} \alpha_{\omega}=\sum_{\substack{\mathbb{X} \\ \mathbb{X} \in \mathcal{P}(\mathbb{D}) \wedge|\mathbb{X}| \leq 2}}\left[\Delta \alpha_{\omega}^{\mathbb{X}}(\mathrm{cc}-\mathrm{pVDZ})-\Delta \alpha_{\omega}^{\mathbb{X}}(\text { aug-cc-pVDZ })\right],
$$

where the subscript on $\Delta$ refers to the order of the incremental expansion in the large basis set. This leads to various schemes for determining the polarizability, as shown in Fig. 7. While the simple shift with $\mu$ corrects for the large offset, it does nothing to improve the breadth of the error distribution. However, use of the incremental scheme yields much narrower Gaussians and thus higher accuracy. When considering the corrected small basis set calculations using eqn (10) and (11) we find an improvement on the second order expansion, and the accuracy improves with increasing computational effort due to the additional terms in the larger basis set. The most accurate results are obtained, of course, for the third order expansion in the aug-cc-pVDZ basis set.

\subsection{Accuracy for specific types of systems}

The significant variation in polarizability errors shown in Fig. 6-and the lack of such variations in the correlation energies-motivates one to consider the characteristics of the molecular systems that might lead to larger errors in the incremental scheme for response properties. We thus classify the molecules in our test set using three criteria: (1) weakly bound systems (13-15, 25, 26, 32-34, and 41-45); (2) quasi-linear/chainlike molecules $(1-8,10,11,16-18,20-22,27,28,30,31,35-40$, and 47); (3) branched/three-dimensional structures (9, 12, 19, 23, 24,29 , and 46). We compare the performance of the incremental scheme for both the dipole polarizability and the correlation energy.

Fig. 8 depicts the error distributions in the polarizability (left) and correlation energy (right) for the weakly bound systems, all of which are optimized structures of dimethylallene (2,3-pentadiene) interacting with one or more molecules of water or chloromethane. Comparing the Gaussian distributions, we find the same trend for the polarizabilities and the energies: the second order for the full set has a rather broad distribution, while the third order is significantly more compact. Considering the subset of weakly interacting systems, we find a relatively compact Gaussian at second order and very sharply peaked Gaussian at third order. Furthermore, the distributions for the full set are in any case significantly broader than those of the subset. Clearly the incremental scheme performs well for the weakly interacting systems considered here, which is unsurprising given the local nature of the interactions in such species. This suggests that the incremental scheme may be particularly valuable for describing the properties of solvated molecules, especially additive properties such as energies and polarizabilities.

The errors in the incremental scheme for quasi-linear molecules are shown in Fig. 9. Unlike the set of weakly bound systems described above, for this subset we find very similar distributions compared to the full set, though the distributions are somewhat narrower at each order for the linear systems. Furthermore, while the correlation energies from the second-order incremental scheme are too small for most of the systems considered here, thus leading 

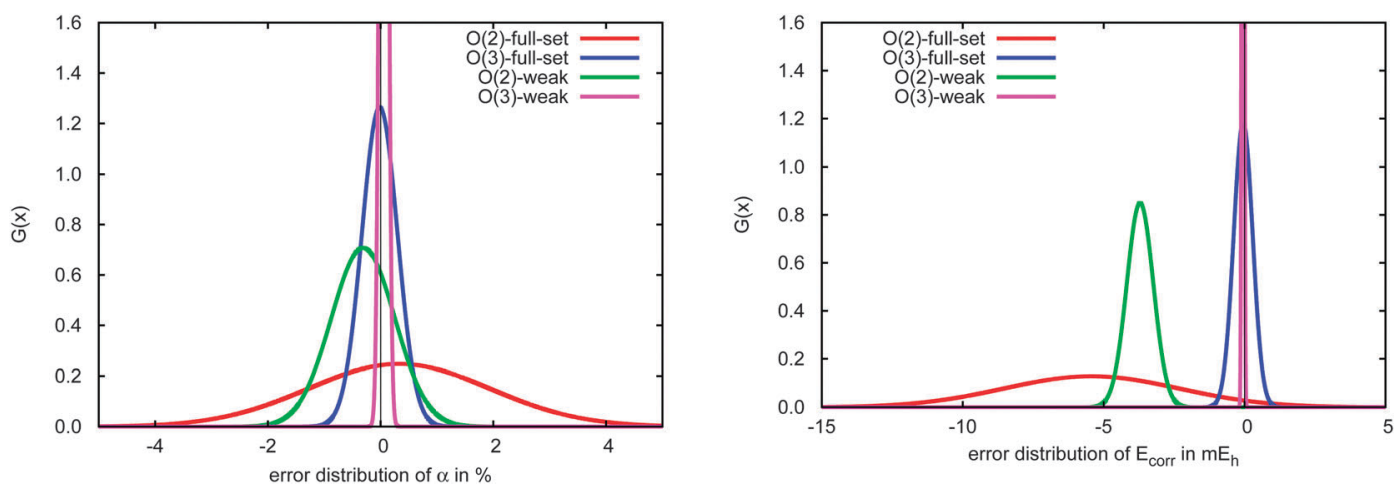

Fig. 8 Analysis of errors in the CCSD/cc-pVDZ dipole polarizabilities (left in \%) and correlation energies (right in $\mathrm{m} E_{\mathrm{h}}$ ) due to the local approximations for weakly bound systems $(13-15,25,26,32-34,41-45)$. The incremental scheme yields somewhat higher accuracy for such systems at both the secondand the third-order as compared to the full set.
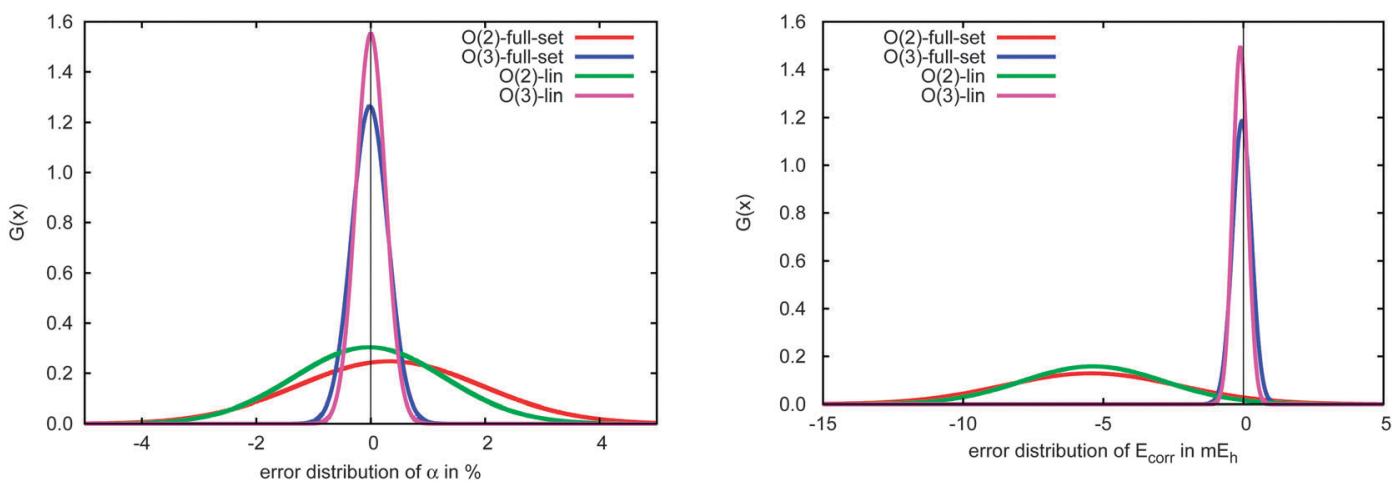

Fig. 9 Analysis of the errors in the CCSD/cc-pVDZ dipole polarizabilities (left in \%) and correlation energies (right in $m E_{h}$ ) due to the local approximations for (quasi)linear systems $(1-8,10,11,16-18,20-22,27,28,30,31,35-40,47)$. The incremental scheme yields similar accuracy for such systems at both the second- and the third-order as compared to the full set.

to Gaussian distributions shifted away from zero (relatively large mean error), errors in the dipole polarizability vary more consistently around zero, leading to smaller (relative) mean errors. At third-order, however, the distributions for both polarizabilities and correlation energies shift much closer to zero. The relatively narrow distributions of errors for linear species arises because the number of interactions between domains increases linearly with the size of the system, leading to a relatively slow accumulation of errors within the incremental scheme.

In Fig. 10 we compare the performance of the incremental scheme for the remaining systems, which contain two- and three-dimensional moieties and thus more strongly interacting domains. These structures are clearly the source of the largest errors arising from the incremental expansion in both polarizabilities and correlation energies, as they yield even broader error distributions than the full test set. This observation, which holds for both the second- and third-order expansions in both polarizabilities and correlation energies, can be explained by the fact that a three-dimensional structure naturally includes more domains that are spatially proximate. Thus, approximations to the interactions between and within a domain, such as those introduced by the incremental scheme, necessarily accumulate a larger number of errors in two- and three-dimensional molecules as compared to a quasi-linear compound or weakly bound complex.

\subsection{Applicability}

As noted earlier, due to the substantial computational effort of the canonical CCSD linear-response method we elected to compute the dipole polarizabilities with the aug-cc-pVDZ basis set for a subset of our 47-molecule test suite, and compound 33-dimethylallene interacting with two water moleculesprovides an example of the computational bottleneck. The convergence of the incremental scheme for the wavelengthdependent CCSD dipole polarizability of this structure is given in Table 4 for four wavelengths $(\lambda=355,436,589$, and $633 \mathrm{~nm})$.

As observed before, the results change significantly when adding diffuse functions, and the cc-pVDZ dipole polarizabilities are about $20 \%$ too small for the second- and thirdorder expansions compared to the aug-cc-pVDZ basis. In addition, we find that the basis set errors are much larger than those arising from local approximations in the incremental scheme. Considering the second-order expansion, for example, we find an maximum error of $1 \%$ in the polarizability when using the cc-pVDZ basis set, falling to $c a .0 .1 \%$ at third-order. We thus may conclude that the incremental scheme can be 

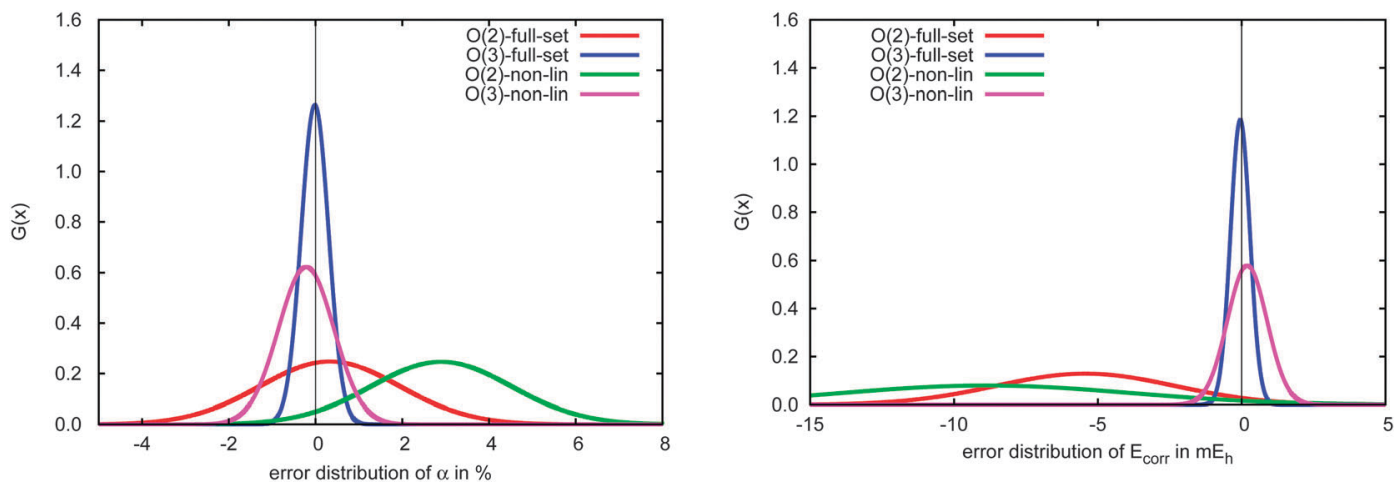

Fig. 10 Analysis of the errors in the CCSD/cc-pVDZ dipole polarizabilities (left in \%) and correlation energies (right in $m E_{h}$ ) due to the local approximations for non-linear systems $(9,12,19,23,24,29,46)$. The incremental scheme yields significantly larger errors at both second- and thirdorder truncation as compared to the full test set.

Table 4 Convergence of the incremental scheme for CCSD dipole polarizabilities for test molecule 33 (dimethylallene interacting with two water molecules) where $\alpha_{\omega}(i)$ denotes the $i$-th order contribution to the polarizability and $\sum \Delta \alpha_{\omega}(i)$ is the sum of all contributions up to order $i$. "Canonical" refers to the untruncated CCSD linear-response result

\begin{tabular}{|c|c|c|c|c|c|c|}
\hline \multicolumn{5}{|l|}{ cc-pVDZ } & \multicolumn{2}{|c|}{ aug-cc-pVDZ } \\
\hline \multirow[b]{2}{*}{ Order $i$} & \multirow{2}{*}{$\frac{\alpha_{\omega}(i)}{\text { a.u. }}$} & \multirow{2}{*}{$\frac{\sum_{i} \Delta \alpha_{\omega}(i)}{\text { a.u. }}$} & \multicolumn{2}{|l|}{ Error } & \multirow{2}{*}{$\frac{\alpha_{\omega}(i)}{\text { a.u. }}$} & \multirow{2}{*}{$\frac{\sum_{i} \alpha_{\omega}(i}{\text { a.u. }}$} \\
\hline & & & a.u. & $\%$ & & \\
\hline \multicolumn{7}{|l|}{$355 \mathrm{~nm}$} \\
\hline 1 & 199.8 & 199.8 & -26.0 & -11.5 & 255.2 & 255.2 \\
\hline 2 & 23.6 & 223.5 & -2.4 & -1.0 & 26.0 & 281.2 \\
\hline 3 & 2.5 & 226.0 & 0.1 & 0.1 & 1.2 & 282.4 \\
\hline Canonical & & 225.9 & & & & \\
\hline \multicolumn{7}{|l|}{$436 \mathrm{~nm}$} \\
\hline 1 & 196.0 & 196.0 & -23.2 & -10.6 & 249.5 & 249.5 \\
\hline 2 & 21.5 & 217.5 & -1.7 & -0.8 & 23.3 & 272.8 \\
\hline 3 & 1.9 & 219.4 & 0.2 & 0.1 & 0.4 & 273.3 \\
\hline Canonical & & 219.2 & & & & \\
\hline \multicolumn{7}{|l|}{$589 \mathrm{~nm}$} \\
\hline 1 & 192.8 & 192.8 & -21.0 & -9.8 & 244.8 & 244.8 \\
\hline 2 & 19.8 & 212.7 & -1.2 & -0.6 & 21.2 & 266.0 \\
\hline 3 & 1.4 & 214.1 & 0.2 & 0.1 & -0.1 & 265.9 \\
\hline Canonical & & 213.9 & & & & \\
\hline \multicolumn{7}{|l|}{$633 \mathrm{~nm}$} \\
\hline 1 & 192.3 & 192.3 & -20.7 & -9.7 & 244.1 & 244.1 \\
\hline 2 & 19.6 & 211.9 & -1.1 & -0.5 & 20.9 & 265.0 \\
\hline 3 & 1.3 & 213.2 & 0.2 & 0.1 & -0.1 & 264.8 \\
\hline Canonical & & 213.0 & & & & \\
\hline
\end{tabular}

used to increase the accuracy of the dipole polarizabilities at the CCSD level of theory, since it makes it feasible to employ significantly larger basis.

\section{Conclusion}

We have presented the first implementation of the incremental scheme for CCSD dipole polarizabilities. The method is fully automatic and makes use of the domain-specific basis set approach. The accuracy of the results has been analyzed using a test suite of 47 molecular systems for which we find that a second-order incremental expansion provides CCSD dipole polarizabilities with a mean error of $0.6 \%$ and a standard deviation of $1.7 \%$. At the third-order level the mean error falls to only $-0.02 \%$ with a corresponding standard deviation of $0.3 \%$. With the proposed approach we are able to compute the CCSD polarizabilities using larger basis sets than is feasible with the canonical approach. Thus, given the strong basis-set dependence of properties such as polarizabilities-particular on diffuse functions-the incremental scheme can be used to increase the total accuracy of the computation of dipole polarizabilities.

In addition, we analyzed which type of molecular structures lead to smaller or larger errors within the incremental scheme. Weakly interacting systems, such as those involved in solvation or those dominated by dispersion interactions, as well as quasilinear structures can be treated with higher accuracy than two- or three-dimensional species. The reason for the differences is associated with the number of strong interactions between and within domains in each type of system, with cage-like compounds yielding the largest errors.

\section{Acknowledgements}

JF thanks the German science foundation (FR2898/4-1) and the Fonds der Chemischen Industrie for financial support. Work at Virginia Tech was supported by grants CHE-1058420 and ACI-1147794 from the U.S. National Science Foundation.

\section{References}

1 R. J. Bartlett, Mol. Phys., 2010, 108, 2905-2920.

2 R. J. Bartlett and M. Musial, Rev. Mod. Phys., 2007, 79, 291-352.

3 R. J. Bartlett, How and why coupled-cluster theory became the pre-eminent method in an ab initio quantum chemistry, in Theory and Applications of Computational Chemistry: The First Forty Years, ed. C. Dykstra et al., Elsevier, New York, 2005, ch. 42, pp. 1191-1221. 
4 T. D. Crawford and H. F. Schaefer, An introduction to coupled cluster theory for computational chemists, in Reviews in Computational Chemistry, ed. K. B. Lipkowitz and D. B. Boyd, VCH Publishers, New York, 2000, ch. 2, vol. 14, pp. 33-136.

5 H. Koch and P. Jørgensen, J. Chem. Phys., 1990, 93, 3333-3344.

6 G. D. Purvis and R. J. Bartlett, J. Chem. Phys., 1982, 76, 1910-1918.

7 K. Raghavachari, G. W. Trucks, J. A. Pople and M. HeadGordon, Chem. Phys. Lett., 1989, 157, 479.

8 H. Koch, O. Christiansen, P. Jørgensen, A. M. S. de Merás and T. Helgaker, J. Chem. Phys., 1997, 106, 1808-1818.

9 H. Koch, H. J. Aa Jensen, P. Jørgensen and T. Helgaker, J. Chem. Phys., 1990, 93, 3345-3350.

10 H. Koch, O. Christiansen, P. Jørgensen and J. Olsen, Chem. Phys. Lett., 1995, 244, 75-82.

11 H. Sekino and R. J. Bartlett, Int. J. Quantum Chem., Symp., 1984, 18, 255.

12 J. F. Stanton and R. J. Bartlett, J. Chem. Phys., 1993, 98, 7029-7039.

13 O. Christiansen, H. Koch, A. Halkier, P. Jørgensen, T. Helgaker and A. S. de Merás, J. Chem. Phys., 1996, 105, 6921-6931.

14 T. B. Pedersen and H. Koch, Chem. Phys. Lett., 1998, 293, 251-260.

15 L. D. Barron, Molecular Light Scattering and Optical Activity, Cambridge University Press, Camridge, UK, 2nd edn, 2004.

16 T. B. Pedersen, H. Koch and K. J. Ruud, Chem. Phys., 1999, 110, 2883-2892.

17 M. Pecul and K. Ruud, Adv. Quantum Chem., 2005, 50, 185-212.

18 T. D. Crawford, Theor. Chem. Acc., 2006, 115, 227-245.

19 T. D. Crawford, M. C. Tam and M. L. Abrams, J. Phys. Chem. A, 2007, 111, 12057-12068.

20 J. F. Stanton and R. J. Bartlett, J. Chem. Phys., 1993, 99, 5178-5183.

21 P. B. Rozyczko, S. A. Perera, M. Nooijen and R. J. Bartlett, J. Chem. Phys., 1997, 107, 6736-6747.

22 P. Rozyczko and R. J. Bartlett, J. Chem. Phys., 1997, 107, 10823-10826.

23 O. Christiansen, C. Hättig and J. Gauss, J. Chem. Phys., 1998, 109, 4745-4757.

24 J. Gauss, O. Christiansen and J. F. Stanton, Chem. Phys. Lett., 1998, 296, 117-124.

25 K. Ruud, T. Helgaker, K. L. Bak, P. Jørgensen and H. J. Jensen, J. Chem. Phys., 1999, 99, 3847-3859.

26 C. Hättig, W. Klopper, A. Köhn and D. P. Tew, Chem. Rev., 2012, 112, 4-74.

27 A. Halkier, T. Helgaker, P. Jørgenson, W. Klopper, H. Koch, J. Olsen and A. K. Wilson, Chem. Phys. Lett., 1998, 286, 243-252.

28 W. Klopper, F. R. Manby, S. Ten-no and E. F. Valeev, Int. Rev. Phys. Chem., 2006, 25, 427.

29 L. Kong, F. A. Bischoff and E. F. Valeev, Chem. Rev., 2012, 112, 75-107.
30 P. Pulay, Chem. Phys. Lett., 1983, 100, 151-154.

31 S. Saebø and P. Pulay, Chem. Phys. Lett., 1985, 113, 13.

32 C. Hampel and H.-J. Werner, J. Chem. Phys., 1996, 104, 6286-6297.

33 M. Schütz, G. Hetzer and H. J. Werner, J. Chem. Phys., 1999, 111, 5691-5705.

34 M. Schütz, J. Chem. Phys., 2000, 113, 9986-10001.

35 H.-J. Werner and M. Schütz, J. Chem. Phys., 2011, 135, 144116.

36 H.-J. Werner, J. Chem. Phys., 2008, 129, 101103.

37 T. B. Adler and H.-J. Werner, J. Chem. Phys., 2011, 135, 144117.

38 T. B. Adler and H.-J. Werner, J. Chem. Phys., 2009, 130, 241101.

39 J. Yang, Y. Kurashige, F. R. Manby and G. K. L. Chan, J. Chem. Phys., 2011, 134, 044123.

40 F. Neese, A. Hansen and D. G. Liakos, J. Chem. Phys., 2009, 131, 064103.

41 D. G. Liakos, A. Hansen and F. Neese, J. Chem. Theory Comput., 2011, 7, 76-87.

42 C. Riplinger and F. Neese, J. Chem. Phys., 2013, 138, 034106.

43 D. G. Fedorov and K. Kitaura, J. Chem. Phys., 2004, 121, 2483-2490.

44 D. G. Fedorov and K. Kitaura, J. Chem. Phys., 2005, 123, 134103.

45 S. R. Pruitt, M. A. Addicoat, M. A. Collins and M. S. Gordon, Phys. Chem. Chem. Phys., 2012, 14, 7752-7764.

46 D. G. Fedorov, T. Nagata and K. Kitaura, Phys. Chem. Chem. Phys., 2012, 14, 7562-7577.

47 Y. Mochizuki, K. Yamashita, T. Nakano, Y. Okiyama, K. Fukuzawa, N. Taguchi and S. Tanaka, Theor. Chem. Acc., 2011, 130, 515-530.

48 W. Yang, Phys. Rev. Lett., 1991, 66, 1438-1441.

49 M. Kobayashi and H. Nakai, J. Chem. Phys., 2009, 131, 114108.

50 M. Kobayashi and H. Nakai, Phys. Chem. Chem. Phys., 2012, 14, 7629-7639.

51 N. Flocke and R. J. Bartlett, J. Chem. Phys., 2004, 121, 10935.

52 T. F. Hughes, N. Flocke and R. J. Bartlett, J. Phys. Chem. A, 2008, 112, 5994-6003.

53 T. F. Hughes and R. J. Bartlett, J. Chem. Phys., 2008, 129, 054105.

54 W. Förner, J. Ladik, P. Otto and J. Cizek, Chem. Phys., 1985, 97, 251.

55 S. Li, J. Shen, W. Li and Y. Jiang, J. Chem. Phys., 2006, 125, 074109.

56 W. Li and P. Piecuch, J. Phys. Chem. A, 2010, 114, 6721-6727.

57 W. Li, P. Piecuch, J. R. Gour and S. Li, J. Chem. Phys., 2009, 131, 114109.

58 W. Li, Y. Guo and S. Li, Phys. Chem. Chem. Phys., 2012, 14, 7854-7862.

59 Z. Rolik and M. Kallay, J. Chem. Phys., 2012, 135, 104111.

60 Z. Rolik, L. Szegedy, I. Ladjánszki, B. Ladóczki and M. Kállay, J. Chem. Phys., 2013, 139, 094105. 
61 M. S. Gordon, D. G. Fedorov, S. R. Pruitt and L. V. Slipchenko, Chem. Rev., 2012, 112, 632-672.

62 T. Korona and H.-J. Werner, J. Chem. Phys., 2003, 118, 3006-3019.

63 T. Korona, K. Pflüger and H.-J. Werner, Phys. Chem. Chem. Phys., 2004, 6, 2059-2065.

64 T. Korona, D. Kats, M. Schütz, T. B. Adler, Y. Liu and H.-J. Werner, Local Approximations for an Efficient and Accurate Treatment of Electron Correlation and Electron Excitations in Molecules, in Linear-Scaling Techniques in Comptuational Chemistry and Physics, ed. R. Zaleśny, M. G. Papadopoulos, P. G. Mezey and J. Leszczynski, Springer, Netherlands, 2011, vol. 13, pp. 345-407.

65 D. Kats, T. Korona and M. Schütz, J. Chem. Phys., 2006, 125, 104106.

66 D. Kats, T. Korona and M. Schütz, J. Chem. Phys., 2007, 127, 064107.

67 K. Freundorfer, D. Kats, T. Korona and M. Schütz, J. Chem. Phys., 2010, 133, 244110.

68 T. D. Crawford and R. A. King, Chem. Phys. Lett., 2002, 366, 611-622.

69 N. J. Russ and T. D. Crawford, Chem. Phys. Lett., 2004, 400, 104-111.

70 N. J. Russ and T. D. Crawford, Phys. Chem. Chem. Phys., 2008, 10, 3345-3352.

71 T. D. Crawford, Reduced-Scaling Coupled-Cluster Theory for Response Properties of Large Molecules, in Recent Progress in Coupled Cluster Methods: Theory and Applications, ed. P. Carsky, J. Pittner and J. Paldus, Springer, Berlin, 2010, ch. 2, vol. 11, pp. 37-55.

72 H. R. McAlexander, T. J. Mach and T. D. Crawford, Phys. Chem. Chem. Phys., 2012, 14, 7830-7836.

73 H. Stoll, Chem. Phys. Lett., 1992, 191, 548-552.

74 H. Stoll, Phys. Rev. B: Condens. Matter Mater. Phys., 1992, 46, 6700-6704.

75 H. Stoll, J. Chem. Phys., 1992, 97, 8449-8454.

76 R. K. Nesbet, Phys. Rev., 1967, 155, 51-55.

77 R. K. Nesbet, Phys. Rev., 1968, 175, 2-9.

78 R. K. Nesbet, Adv. Chem. Phys., 1969, 14, 1-34.

79 K. Doll, M. Dolg, P. Fulde and H. Stoll, Phys. Rev. B: Condens. Matter Mater. Phys., 1995, 52, 4842-4848.

80 K. Doll, M. Dolg and H. Stoll, Phys. Rev. B: Condens. Matter Mater. Phys., 1996, 54, 13529-13534.

81 K. Doll, M. Dolg, P. Fulde and H. Stoll, Phys. Rev. B: Condens. Matter Mater. Phys., 1997, 55, 10282-10287.

82 S. Kalvoda, M. Dolg, H.-J. Flad and P. Fulde, Phys. Rev. B: Condens. Matter Mater. Phys., 1998, 57, 2127-2133.

83 K. Rosciszewski, B. Paulus, P. Fulde and H. Stoll, Phys. Rev. B: Condens. Matter Mater. Phys., 1999, 60, 7905-7910.

84 K. Rosciszewski, B. Paulus, P. Fulde and H. Stoll, Phys. Rev. B: Condens. Matter Mater. Phys., 2000, 62, 5482-5488.

85 H. Stoll, B. Paulus and P. Fulde, J. Chem. Phys., 2005, 123, 144108. 86 C. Müller, B. Herschend, K. Hermansson and B. Paulus, J. Chem. Phys., 2008, 128, 214701.

87 I. Schmitt, K. Fink and V. Staemmler, Phys. Chem. Chem. Phys., 2009, 11, 11196-11206.
88 C. Müller, K. Hermansson and B. Paulus, Chem. Phys., 2009, 362, 91-96.

89 V. Staemmler, J. Phys. Chem. A, 2011, 115, 7153-7160.

90 C. Müller, D. Usvyat and H. Stoll, Phys. Rev. B: Condens. Matter Mater. Phys., 2011, 83, 245136.

91 S. Kalvoda, B. Paulus, M. Dolg, H. Stoll and H.-J. Werner, Phys. Chem. Chem. Phys., 2001, 3, 514-522.

92 B. Paulus, Int. J. Quantum Chem., 2004, 100, 1026.

93 J. Friedrich, E. Perlt, M. Roatsch, C. Spickermann and B. Kirchner, J. Chem. Theory Comput., 2011, 7, 843-851.

94 C. Spickermann, E. Perlt, M. Domaros, M. Roatsch, J. Friedrich and B. Kirchner, J. Chem. Theory Comput., 2011, 7, 868-875.

95 E. Perlt, J. Friedrich, M. Domaros and B. Kirchner, ChemPhysChem, 2011, 12, 3474-3482.

96 I. Polenz, F. G. Schmidt, J. Friedrich, I. Tchernook and S. Spange, Macromol. Chem. Phys., 2013, 214, 1473-1483.

97 J. Zhang and M. Dolg, J. Chem. Theory Comput., 2013, 9, 2992-3003.

98 J. Zhang, N. Heinz and M. Dolg, Inorg. Chem., 2014, 53, 7700-7708.

99 M. Modl, M. Dolg, P. Fulde and H. Stoll, J. Chem. Phys., 1997, 106, 1836-1846.

100 J. Friedrich, M. Hanrath and M. Dolg, J. Phys. Chem. A, 2008, 112, 8762-8766.

101 E. N. Voloshina and B. Paulus, J. Chem. Theory Comput., 2014, 10, 1698-1706.

102 J. Yang and M. Dolg, J. Chem. Phys., 2007, 127, 084108.

103 J. Friedrich, S. Coriani, T. Helgaker and M. J. Dolg, Chem. Phys., 2009, 131, 154102.

104 K. Hald, F. Pawłowski, P. Jørgenson and C. Hättig, J. Chem. Phys., 2003, 118, 1292.

105 O. Christiansen, J. Gauss and J. F. Stanton, Chem. Phys. Lett., 1998, 292, 437.

106 O. Christiansen, P. Jørgensen and C. Hättig, Int. J. Quantum Chem., 1998, 68, 1-52.

107 P. Jørgensen and J. Simons, Second Quantization-Based Methods in Quantum Chemistry, Academic Press, New York, 1981.

108 R. Kobayashi, H. Koch and P. Jørgensen, Chem. Phys. Lett., 1994, 219, 30-35.

109 J. Friedrich, M. Hanrath and M. Dolg, J. Chem. Phys., 2007, 126, 154110.

110 J. Friedrich, M. Hanrath and M. Dolg, J. Phys. Chem. A, 2007, 111, 9830-9837.

111 J. Friedrich, M. Hanrath and M. Dolg, Chem. Phys., 2007, 338, 33-43.

112 J. M. Foster and S. F. Boys, Rev. Mod. Phys., 1960, 32, 300.

113 J. Friedrich, M. Hanrath and M. Dolg, Z. Phys. Chem., 2010, 224, 513-525.

114 A. D. Becke, Phys. Rev. A: At., Mol., Opt. Phys., 1988, 38, 3098-3100.

115 S. H. Vosko, M. Wilk and M. Nusair, Can. J. Phys., 1980, 58, 1200.

116 J. P. Perdew, Phys. Rev. B: Condens. Matter Mater. Phys., 1986, 33, 8822-8824. 
117 A. Schäfer, H. Horn and R. I. Ahlrichs, J. Chem. Phys., 1992, 97, 2571-2577.

118 J. Friedrich and J. Hänchen, J. Chem. Theory Comput., 2013, 9, 5381-5394.

119 A. D. Becke, J. Chem. Phys., 1993, 98, 5648.

120 C. Lee, W. Yang and R. G. Parr, Phys. Rev. B: Condens. Matter Mater. Phys., 1988, 37, 785-789.

121 P. J. Stephens, F. J. Devlin, C. F. Chabalowski and M. J. Frisch, J. Phys. Chem., 1994, 98, 11623-11627.

122 T. H. Dunning Jr., J. Chem. Phys., 1989, 90, 1007-1023.
123 R. A. Kendall, T. H. Dunning Jr. and R. J. Harrison, J. Chem. Phys., 1992, 96, 6796-6806.

124 D. E. Woon and T. H. Dunning, J. Chem. Phys., 1993, 98, 1358.

125 J. M. Turney, et al., Wiley Interdiscip. Rev.: Comput. Mol. Sci., 2012, 2, 556-565.

126 J. Friedrich and K. Walczak, J. Chem. Theory Comput., 2013, 9, 408-417.

127 W. J. Hehre, R. Ditchfield and J. A. Pople, J. Chem. Phys., 1972, 56, 2257. 